\title{
Research highlights from the 2017 ERS International Congress: airway diseases in focus
}

\author{
Cecilia Andersson ${ }^{1}$, Sara J. Bonvini ${ }^{1}$, Peter Horvath ${ }^{2}$, Eduardo Marquez ${ }^{3,4}$, \\ Imran Satia ${ }^{5}$, Paul Kirkham ${ }^{6}$, Florence Schleich ${ }^{7}$, Marco Idzko ${ }^{8}$, \\ Reinoud Gosens ${ }^{9}$, Jose Luis Lopez-Campos ${ }^{4}$, Apostolos Bossios ${ }^{10,11,12}$, \\ Omar Usmani ${ }^{1}$, Antonio Spanevello ${ }^{13}$, Ian M. Adcock ${ }^{1}$ and \\ Alexander G. Mathioudakis ${ }^{5}$
}

Affiliations: ${ }^{1}$ National Heart and Lung Institute, Imperial College, London, UK. ${ }^{2}$ Dept of Pulmonology, Semmelweis University, Budapest, Hungary. ${ }^{3}$ Unidad Médico-Quirúrgica de Enfermedades Respiratorias, Instituto de Biomedicina de Sevilla (IBiS), Campus Hospital Universitario Virgen del Rocío and Universidad de Sevilla, Seville, Spain. ${ }^{4}$ Centro de Investigación Biomédica en Red Enfermedades Respiratorias (CIBERES) and Instituto de Salud Carlos III (ISCIII), Madrid, Spain. ${ }^{5}$ Division of Infection, Immunity and Respiratory Medicine, University of Manchester, Manchester, UK. ${ }^{6}$ Dept of Biomedical Sciences, University of Wolverhampton, Wolverhampton, UK. ${ }^{7}$ Dept of Respiratory Medicine, Centre Hospitalier Universitaire (CHU) de Liège and Research Group GIGA 13, University of Liège, Liège, Belgium. ${ }^{8}$ Dept of Pneumology, Medical University of Vienna, Vienna, Austria. ${ }^{9}$ Dept of Molecular Pharmacology, University of Groningen, Groningen, The Netherlands. ${ }^{10}$ Dept of Respiratory Medicine and Allergy, Karolinska University Hospital, Stockholm, Sweden. ${ }^{11}$ Dept of Medicine, Huddinge, Karolinska Institutet, Stockholm, Sweden. ${ }^{12}$ Centre for Allergy Research, Karolinska Institutet, Stockholm, Sweden. ${ }^{13}$ Università degli Studi dell'Insubria and Fondazione S. Maugeri, Varese, Italy.

Correspondence: A.G. Mathioudakis, Division of Infection, Immunity and Respiratory Medicine, University of Manchester, Southmoor Road, Manchester, M9 7AL, UK. E-mail: alexander.mathioudakisamanchester.ac.uk

ABSTRACT For another year, high-quality research studies from around the world transformed the annual ERS International Congress into a vivid platform to discuss trending research topics, to produce new research questions and to further push the boundaries of respiratory medicine and science. This article reviews only some of the high-quality research studies on asthma, chronic obstructive pulmonary disease (COPD), bronchiectasis and chronic cough that were presented during the congress through the Airway Diseases Assembly (ERS Assembly 5) and places them into the context of current knowledge and research challenges.

@ERSpublications

Members of the @ERStalk Airway Diseases Assembly discuss clinical highlights from \#ERSCongress $2017 \mathrm{http}: / /$ ow.ly/G51Y30i7fMR

Cite this article as: Andersson C, Bonvini SJ, Horvath P, et al. Research highlights from the 2017 ERS International Congress: airway diseases in focus. ERJ Open Res 2018; 4: 00163-2017 [https:// doi.org/10.1183/23120541.00163-2017].

Received: Dec 182017 | Accepted after revision: Jan 112018

Conflict of interest: R. Gosens reports receiving grants from Chiesi Farmaceutici, Boehringer Ingelheim and Aquilo BV, outside the submitted work. I. Satia reports receiving a BMA James Trust Award, personal fees from educational talks for general practitioners, travel awards and an ERS Respire 3 Fellowship Award, outside the submitted work. O. Usmani reports receiving personal fees from AstraZeneca, Boehringer Ingelheim, Chiesi, GlaxoSmithKline, NAPP, Mundipharma, Sandoz, Cipla, Takeda, Zentiva and Trudell Medical, and grants from AstraZeneca, Boehringer Ingelheim, Chiesi, Prosonix, Edmond Pharma, Cipla, NAPP, Mundipharma, Sandoz and Aerocrine, outside the submitted work.

Copyright $\odot$ ERS 2018. This article is open access and distributed under the terms of the Creative Commons Attribution Non-Commercial Licence 4.0. 


\section{Asthma}

Asthma is one of the most common inflammatory diseases globally yet it has been defined by quite broad definitions, such as breathlessness and wheezing, that are associated with variable airflow obstruction and inflammation within the lungs [1]. It becomes increasingly clear that asthma shows a high degree of heterogeneity [2]. Nevertheless, treatment strategies for asthma are largely non-specific, commonly including glucocorticosteroids and bronchodilators. Although most patients respond well to this therapy, a remaining group of asthmatic patients does not respond to the available treatments, accounting for a great part of the economic and health burden of the disease [3]. Furthermore, diagnosis and/or treatment of unresponsiveness to therapy or severity of asthma often does not take into account the various underlying pathological processes, mostly due to the limited information available on how to correlate clinical phenotypes to biological endotypes $[4,5]$.

In a study by BIGLER et al. [6], nearly 1700 genes were found to be differently expressed in blood samples from severe asthma patients compared to non-asthmatic individuals. In asthmatic patients, pathway analysis showed specific immune cell patterns such as upregulation of genes associated with eosinophils and neutrophils and decreased expression of genes involved in blood-lymphocyte development and haematopoietic progenitor cells. Interestingly, cluster analysis showed subgroups within the severe asthma group who differed in their response to treatment with corticosteroids [6]. At this year's congress, AfFLECK et al. [7] presented a sequential study comparing blood transcriptomic profiles of adults and children from the U-BIOPRED study with the aim of investigating if a similar dysregulation would be seen in children with asthma. They reported that while the school-aged children showed some differentially expressed gene (DEG) overlap with the adults they were distinct in many pathways, indicating that childhood and adult asthma may be mechanistically different [7].

Indeed, age of disease onset is a significant discriminating factor between different asthma phenotypes and an important factor in severe asthma [8]. In a recent study by HeKKING et al. [9], gene set variation analysis was used to identify gene profiles associated with adult-onset severe asthma. This study compared adult-onset with childhood-onset severe asthmatics from the U-BIOPRED cohort using samples from nasal and bronchial brushings, induced sputum and endobronchial biopsies. Gene signatures related to airway inflammation characterised by eosinophils, mast cells and innate lymphocyte cells (ILCs) of Group 3 (ILC3s) were enriched in adult-onset severe asthma. The study by HeKKING et al. [9] also showed that in childhood-onset severe asthma, signatures associated with lung damage were more pronounced. This is interesting as it has been proposed that impaired epithelial damage repair mechanisms are important drivers in asthma development [10]; however, very little is known regarding the natural origin of asthma development. Furthermore, PAVLIDIs et al. [11] presented a follow-up study from the U-BIOPRED group at this year's European Respiratory Society (ERS) Congress which aimed to explore the enrichment of ILC1, ILC2 and ILC3 activation signatures in asthmatic transcriptomic samples. From this study, they conclude that a neutrophilic severe asthma phenotype was characterised by Group 1 ILC activation, associated with inflammasome, interferon- $\gamma$ (IFN- $\gamma$ ) and tumour necrosis factor (TNF), which may reflect an antimicrobial response and contribute to chronic inflammation and remodelling in severe asthma.

Respiratory syncytial virus (RSV) and rhinovirus infections are major causes of bronchiolitis and pneumonia and are strongly associated with asthma exacerbations $[12,13]$. Wheezing in response to viral infections correlates to asthma development in children $[14,15]$ and, while the majority children who wheeze upon viral infection recover without any enduring effects, a proportion of children with wheeze will develop asthma later in life [16, 17]. Despite strong evidence from longitudinal studies, it is not known how viral infections during early childhood are connected to the development of asthma $[16,17]$. However, two concepts have emerged to explain why asthmatic patients are more sensitive to viral infections: 1) an exaggerated Type 2 T-helper cell (Th2) immune response towards viruses with increased levels of interleukin (IL)-4, IL-5 and IL-13 cytokines, and 2) a deficient anti-viral response primarily explained by impaired production of Type I and Type III IFNs [12]. Several in vivo and in vitro studies in human airway cells and murine models have shown a deficiency in IFN production in response to viral infection, a trend which is especially pronounced in severe asthmatic disease (see EDwARDs et al. [18]). Furthermore, the risk of developing asthma is increased with frequent viral-induced bronchiolitic episodes in combination with allergic sensitisation to airborne allergens [19]. RuBNER et al. [20] showed in a recent study that the age of sensitisation (age $<1$ year) and rhinovirus infection (but not RSV) was important for asthma development by adolescence. It has furthermore been reported that rhinovirus causes amplified release of double stranded DNA in individuals with asthma undergoing rhinovirus-induced asthma exacerbations and that the levels correlated with both symptom severity during exacerbation and rhinovirus-induced release of Type 2 cytokines [21]. In a study by DANOv et al. [22], which was presented at this year's ERS Congress, the impact of an asthmatic background on the anti-viral and pro-inflammatory immune response was investigated in precision-cut lung slices (PCLS) of house dust 
mite (HDM)-sensitised mice. The authors showed that the HDM-induced allergic inflammation was maintained ex vivo in the PCLS and led to a reduced immune response to rhinovirus infection, as shown by decreased secretion of IFN- $\gamma$, IFN- $\gamma$-inducible protein 10 (IP-10) and IL-17A in response to HRV1B infection. Interestingly, Th2 cytokine IL-4 was further induced in rhinovirus-infected PCLS of HDM-sensitised mice.

A compelling amount of data now points towards a miscommunication between the innate and adaptive immune response towards viruses in asthmatic patients. Recent data has shown that, upon damage, epithelial cells release the alarmin IL-33 and data from genome-wide association studies has implicated IL-33 in the pathobiology of allergic diseases [23, 24]. At the ERS Congress this year there were several abstracts related to the role of IL-33 in asthma pathology (see RAVANETTI et al. [25], HACCURIA et al. [26] and Perkins et al. [27]). Higher levels of IL-33 have been found in subjects with severe asthma when compared with healthy controls [28]. The increased levels of IL-33 might be important since it can promote both innate and adaptive immune responses and can influence the cross-talk between these two arms of immune defense [29, 30]. In line with this, LYNCH et al. [31, 32] reported that IL-33 can suppress innate anti-viral immune defense which in turn results in increased viral burden, airway smooth muscle hyperplasia and Th2 inflammation. A sequential study presented by Uрнам et al. [33] was aimed at examining the mechanisms involved and especially the two chains of the IL-33 receptor (IL-1 receptor like 1 (IL1RL, also known as ST2) and IL-1 receptor accessory protein (IL1RAP)). Peripheral blood mononuclear cells (PBMC) obtained from mild/moderate asthmatics and healthy donors were exposed to IL-33 and activated with rhinovirus. In asthmatic patients, rhinovirus alone or in combination with IL-33 induced significantly greater upregulation of ST2 mRNA expression than in healthy participants but had no effect on IL1RAP expression. The results were confirmed using flow cytometry and the increase was due to enhanced ST2 expression on ILC2s and T-lymphocytes. These findings suggest there is dysregulation of ST2 in asthma and provide a mechanism by which rhinovirus infections might exacerbate Type 2 inflammation.

Taken altogether, asthma is a very heterogeneous disease with specific gene patterns that, with increasing knowledge, will enable a more thorough stratification of asthmatic phenotypes. It is now vital that these phenotypes be linked to biological endotypes, where studies of the effects of biological treatments will be of great importance. The aforementioned studies further stress the notion that the complex interplay between viral infection and allergen exposure is important for the onset and progression of asthmatic disease. Difficult-to-treat patients that are unresponsive to conventional therapy would most likely benefit greatly from a thorough phenotyping and endotyping of their pathophysiology, with the ultimate goal of administering more specialised and personalised medication.

\section{Chronic obstructive pulmonary disease}

Pharmacological treatment of chronic obstructive pulmonary disease (COPD) aims to reduce symptoms, frequency and severity of exacerbations, to improve exercise tolerance and health status, and also to delay disease progression and death [34]. While there is still no conclusive evidence from clinical trials indicating that any of the existing COPD medications modify disease progression, there is fast-accumulating evidence on how to optimise symptom control and exacerbation prevention.

First, several trials assessing triple therapy, inclusive of a long-acting $\beta$-2 agonist, a long-acting antimuscarinic and an inhaled corticosteroid in a single device were presented. The FULFIL trial compared the triple combination of fluticasone furoate, umeclidinium and vilanterol against the combination of budesonide and formoterol in a 24-week randomised trial involving 1810 patients. It demonstrated the superiority of the triple combination with regards to spirometry, frequency of exacerbations, frequency of pneumonias, quality of life (QoL) and time to clinically important deterioration (CID, a composite measure of lung function, health status and exacerbation). The median time to first CID was 169 days for the triple combination versus 31 days for the long-acting $\beta$-agonist (LABA)/inhaled corticosteroid (ICS) combination [35, 36]. However, this study can be criticised for comparing a triple combination versus an ICS/LABA combination, rather than a single dose triple combination versus separate devices, which would have been more informative. Such an intervention was evaluated by the TRINITY trial [37] which compared the fixed triple combination of beclometasone dipropionate, formoterol fumarate and glycopyrronium bromide against the open triple combination of beclometasone dipropionate and formoterol fumarate plus tiotropium, or tiotropium alone, in a 52-week randomised controlled trial involving 2691 patients. The fixed triple combination was found to be superior to the long-acting muscarinic antagonist (LAMA) and to be non-inferior to the open triple combination with regards to frequency of moderate-to-severe exacerbations, time to first CID, spirometry and adverse effects [38]. Use of triple combination therapy is currently only recommended for Global Initiative for Chronic Obstructive Lung Disease (GOLD) Group D patients under current GOLD guidelines [34]. 
However, a post hoc analysis of the TRINITY and TRILOGY [39] trials this year has assessed the impact of fixed triple combination therapy versus LABA/ICS or LAMA therapy in Group B patients and found a significant decrease in the frequency of exacerbations [40].

Dual bronchodilation has also received a lot of attention this year. The FLAME trial, a 52-week trial involving 3362 patients with COPD and a history of at least one moderate or severe exacerbation during the preceding year, compared a fixed combination of indacaterol/glycopyrronium (LABA/LAMA) against salmeterol/fluticasone (LABA/ICS) and demonstrated the superiority of the LABA/LAMA combination versus the LABA/ICS combination with regards to exacerbations, lung function and QoL [41]. Several post-hoc analyses from the FLAME trial were presented this year, evaluating more domains and confirming the superiority of the LABA/LAMA combination. The number needed to treat (NNT) to avoid a moderate or severe COPD exacerbation (per year) was estimated to be 4.76 patients with dual bronchodilation. In addition, the NNT to keep a patient with at least one exacerbation in the preceding year free of exacerbations was 15 [42]. Indacaterol/glycopyrronium was also shown to be useful in reducing the risk of $\mathrm{CID}$, defined as a decrease of $100 \mathrm{~mL}$ in forced expiratory volume in $1 \mathrm{~s}(\mathrm{FEV} 1)$ or an increase of four points on the St George's Respiratory Questionnaire (SGRQ). Indacaterol/glycopyrronium reduced the risk of first and sustained CID by approximately $30 \%$ versus salmeterol/fluticasone, as presented by ANZUETO et al. [43]. Further analyses confirmed the safety of dual bronchodilation as it was associated with fewer respiratory-related adverse events of special interest, including but not limited to pneumonia, compared with salmeterol/fluticasone [44]. The cost effectiveness of indacaterol/ glycopyrronium compared to treatment with salmeterol/fluticasone was also assessed in a real-life context, in the ARCTIC Swedish cohort study of 18,586 subjects over 3 years. Dual bronchodilation was associated with lower total direct costs (a saving of 732 euro per patient) and better outcomes ( 0.008 quality-adjusted life-years and 0.005 life-years gained).

The ACLIFORM and AUGMENT trials compared another dual bronchodilator (aclidinium/formoterol) against its monocomponents or a placebo, in 3394 maintenance treatment naïve patients with moderate or severe COPD over a 24-week period and in a double-blinded fashion. Pooled studies from these trials have demonstrated the superiority of aclidinium/formoterol versus its monocomponents or a placebo with regards to symptoms, QoL, frequency of exacerbations and use of relief medications [45, 46]. ACTIVATE, an 8-week trial of 267 patients also found that aclidinium/formoterol significantly improved physical activity compared to a placebo at 4 and 8 weeks [47].

The use of a fixed-dose dual bronchodilator was also evaluated in 4117 COPD patients under real-life conditions in the DACCORD study [48]. Patients were recruited following a change in their maintenance treatment by their clinicians (as indicated by a non-interventional study) and the exacerbation frequency of patients who received a LABA/LAMA combination versus other treatments was compared [49]. Dual bronchodilation was correlated with significantly fewer exacerbations amongst those patients who had at least one exacerbation in the 6 months preceding recruitment.

Finally, in an interesting animal study, Gosset et al. [50] assessed the effect of prophylactic administration of flagellin, a toll-like receptor 5 (TLR5) agonist, in mice with COPD. Mice treated with flagellin were found to have significantly lower bacterial load in bronchoalveolar lavage (BAL), suggesting a potential protective effect of flagellin against bacterial infection or colonisation. This opens a new therapeutic line in terms of the stimulation of the innate immune response to prevent COPD exacerbations.

Overall, data are fast accumulating which support the effectiveness of bronchodilators, inhaled steroids and combination therapies, which clearly improve the health status and QoL of patients while decreasing the frequency of exacerbations. Unfortunately, there are still significant challenges ahead as none of the available treatments can halt or delay disease progression or prolong survival. In addition, our expanding understanding of COPD heterogeneity and clinical phenotypes has only now started to be translated into the development of better, targeted treatments.

\section{Novel monoclonal antibodies in asthma and COPD}

Asthma and COPD are common chronic inflammatory airways diseases that affect an estimated 700 million people worldwide [51, 52]. Despite taking high dose inhaled and often daily oral steroid treatments, approximately $5-10 \%$ of patients with asthma have severe asthma [53] and between $30-40 \%$ of patients with COPD have recurrent moderate to severe exacerbations on inhaled triple therapy [54], even if there is an increasing body of evidence for positive results in patients' QoL. This results in a high burden of disease on QoL and employment, as well as pressure on healthcare services. Over the last 20 years, there has been a focus on treating uncontrolled patients by understanding the underlying pathological mechanisms which lead to such complex heterogeneous airways diseases. Mechanistic studies have highlighted the role of Type 2 inflammatory cytokines (IL-4, IL-5, IL-9 and IL-13) and airway eosinophils 
as important biomarkers to target in asthma; however, only recently has evidence emerged showing the limited benefits of such a targeted strategy in COPD [55]. A potential limitation in studies targeting IL-5 or eosinophils is that such drugs largely benefit patients who demonstrate an eosinophilic phenotype, hence excluding a large proportion of subjects. However, novel data has shown the importance of inflammatory cytokines secreted from the airway epithelium in orchestrating and activating Type 2 inflammation [56] and Group 2 ILCs (ILC2s) [57]. These are collectively known as the epithelial "alarmins" and currently include IL-33, IL-25 and thymic stromal lymphopoietin (TSLP). The latter has emerged as an important target for asthma, irrespective of allergic or eosinophilic status. Here we focus on data presented at the ERS International Congress 2017 for tezepelumab (in patients with uncontrolled asthma) and mepolizumab (for eosinophilic COPD), as they represent an advancement in both our current understanding and our future treatment options.

TSLP is produced from the airway epithelium in response to a wide variety to external stimuli, such allergens and viruses, and has the ability to act as an "epithelial alarmin" by activating Type 2 inflammation via activation of dendritic cells, ILC2s, T-cells and B-cells, resulting in an increase in airway inflammatory cytokines, particularly IL-4, IL-5 and IL-13. An earlier proof of concept study showed the efficacy of tezepelumab in reducing the early and late asthmatic responses in an allergen challenge model, with profound reductions in both serum and sputum eosinophils [56]. However, most asthma exacerbations occur due to viral exacerbations and all patients in the allergen challenge model were steroid naïve and well controlled. As such, the rationale for this larger multicentre, placebo-controlled, double-blind follow up study was to test the efficacy of tezepelumab in a much broader population of uncontrolled moderate to severe asthma patients unselected for serum eosinophils [58]. Over a 52-week period, tezepelumab (70 mg or $210 \mathrm{mg}$ every 4 weeks, or $210 \mathrm{mg}$ every 2 weeks) reduced exacerbation rates by $61 \%, 71 \%$ and $66 \%$ more than a placebo, respectively, and, most importantly, this improvement was seen irrespective of blood eosinophil count at baseline. There were significant improvements in pre-bronchodilator $\mathrm{FEV}$, asthma control and reductions in exhaled nitric oxide fraction $(\mathrm{FeNO})$ and serum IgE. This suggests that the rationale of inhibiting the inflammatory cascade higher up the pathway is effective at augmenting a wider range of Type 2 inflammatory mediators irrespective of allergic or eosinophil status. These findings make the results of this study different to those observed previously (targeting IL-5 or its receptor subunit only). The only caveats to this study were that the majority of patients were recruited from Eastern Europe and Ukraine, $90 \%$ were Caucasian and there was a large placebo effect. Further Phase 3 studies in different healthcare settings and with broader ranges of ethnicities would add value to these initial results.

Although the targeting of eosinophils has most often been associated with treating asthma, there have been important observations to suggest a role in COPD. The ECLIPSE study showed that nearly $40 \%$ of patients had persistently raised serum eosinophils $(\geqslant 2 \%)$, which was associated with an increased risk of exacerbations, but were highly responsive to corticosteroids [59]. However, the side-effects profile, particularly the increased risk of pneumonia, has more recently led to a renewed emphasis on steroid reduction and alternative treatment strategies. The rationale to this study was rooted in observing an association between lower exacerbation rates than placebo in two mepolizumab in severe asthma studies with some features of COPD (the MENSA and DREAM studies). The objective of this study was to evaluate the efficacy and safety of subcutaneous mepolizumab, as compared with placebo, as an additional treatment to inhaled triple therapy in those COPD patients with an eosinophilic phenotype and a history of moderate or severe exacerbations [60]. An eosinophilic phenotype was defined as $\geqslant 150$ cells $\cdot \mathrm{mm}^{-3}$ at screening or $\geqslant 300$ cells $\cdot \mathrm{mm}^{-3}$ in the previous year and moderate exacerbations were defined as $\geqslant 2$ episodes requiring steroids \pm antibiotics (and as severe if leading to hospitalisation). The data presented demonstrated an $18 \%$ reduction in combined moderate and severe exacerbations only in those patients defined as having an eosinophilic phenotype, with the greatest effect seen in those with the highest eosinophil counts. However, no difference was found in hospitalisations, emergency department visits, FEV1 or QoL endpoints, nor is there any data currently available for the possibility of steroid dose reduction as has been seen in asthma. The implication of these results is to potentially use serum eosinophils as a biomarker to identify those most likely to benefit from such immune therapy. However, this needs to be balanced with the fact that there was a large improvement in exacerbations in the placebo group likely related to improved adherence during the clinical trial. This is not unusual for most trials in COPD and asthma but raises the question of the need for such expensive treatment in a resource-stretched healthcare system.

Overall, studies involving monoclonal antibodies in patients with asthma and now COPD have improved our understanding of airway pathobiology; however, applicability, long term safety and cost effectiveness for a large (and broader) population will ultimately dictate eligibility and access to such treatments. 


\section{Non-cystic fibrosis bronchiectasis}

Bronchiectasis is a disease accompanied by irreversible enlargement of the airways and is characterised by ineffective mucociliary clearance and excessive inflammation. The inflammation is not just excessive it is also ineffective, as it causes airway destruction and distortion which leads to ineffective mucus clearance. Ineffective mucus clearance in turn leads to bacterial colonisation, which itself enhances inflammation-the so called "vicious cycle" of bronchiectasis. Pseudomonas bacteria frequently colonise the airways of patients with bronchiectasis and this is associated with exacerbations and disease severity. This year, Chalmers et al. [61] further evaluated the impact of Pseudomonas colonisation on the outcomes of bronchiectasis using data from the Facilitating Research Into Existing National Datasets (FRIENDS) cohort, a historical dataset integrating national registries of bronchiectasis from the past decade with the intention of giving a clear picture of bronchiectasis management and outcomes and including 2596 patients with high-resolution computed tomography (HRCT) confirmed bronchiectasis. Researchers found Pseudomonas colonisation to be associated with worse symptoms, radiologic severity and more frequent exacerbations (median: three per year). Importantly, they also demonstrated that colonised patients were at twice as high a risk of death.

Furthermore, SMITH et al. [62] identified a novel biomarker of disease severity starting from the observation that most patients with bronchiectasis are women. More specifically, they found high levels of pregnancy zone protein (PZP, an oestrogen-regulated antiprotease) in the sputum of patients with bronchiectasis and explored its prognostic value in 80 patients. PZP was strongly correlated with bronchiectasis severity index, SGRQ, level of breathlessness and Pseudomonas infection.

At the ERS Congress, several high quality studies focused on bronchiectasis management, which is complex and needs to cover the management of both acute exacerbations and stable disease (including exacerbation prevention). During stable disease, control of microbial load decreases inflammation and mucus production and delays subsequent exacerbations. Inhaled antibiotic therapy includes colistin and gentamycin, and there are ongoing trials with ciprofloxacin and levofloxacin. Indeed, FoeHLICH et al. [63] reported in Milan on a post-hoc analysis of two 48-week trials involving 148 participants colonised by Pseudomonas, which demonstrated that long-term, intermittent use of ARD-3150, a combination of liposome-encapsulated and free ciprofloxacin, significantly decreased the frequency of pulmonary exacerbation in bronchiectasis patients, regardless of the Pseudomonas' susceptibility to ciprofloxacin.

Macrolide antibiotics also possess potent anti-inflammatory effects [64]. In cases of rhinovirus infection, macrolides increase the release of IFN and decrease viral load [65]. Many multicentre trials have investigated macrolide use in bronchiectasis and, while they significantly decrease exacerbation rate and increase the time to first exacerbation, they do not influence FEV1. There is however an improvement in QoL based on the SGRQ [66-70]. Macrolides suppress systemic inflammation and C-reactive protein (CRP) decreases along with sputum and peripheral neutrophil counts. If exacerbation rate is more than three per year then the use of macrolides is beneficial. In accordance with these results MERTSCH et al. [71] found that $33 \%$ of patients in a German cohort were on azithromycin maintenance therapy, while DHAR et al. [72] showed that $55.1 \%$ of patients who were on antibiotic treatment in India received low-dose macrolides (31\% with azithromycin).

The role of long-term administration of antibiotics in patients with bronchiectasis and frequent exacerbation is indisputable. However, further studies are required to compare the efficacy and safety of different treatment regimes (different antibiotics, route of administration and frequency etc.). Importantly, bronchial hygiene and mucolytic treatments are effective and safer and need to be prioritised.

\section{Chronic cough}

Cough is the primary reason for patients to visit a physician [73] and yet it remains an unmet medical need. Chronic cough, which can occur as a result of respiratory disease but can also be idiopathic in nature [74] can severely lower the QoL of sufferers [75]. In the year preceding March 2013, over \$156 million was spent in the UK on cough medications [76] despite the fact that they have been shown to be ineffective. Therefore, there is an urgent requirement for new, safe and effective therapies, and the clinical problem of cough was highlighted at the ERS Congress where there was a "meet the expert" seminar on the topic. At this event, Professor Alyn Morice talked clinicians through the underlying mechanisms, diagnosis and treatment of patients with refractory chronic cough.

Cough is a reflex event triggered following activation of airway sensory nerves housed within the vagus nerve [77]. Sensory nerve terminals are distributed throughout the airways and express a number of ion channels and receptors that could represent novel targets for treatment. To date, the only ion channel antagonist that has shown efficacy in refractory chronic cough is P2X2/3 antagonist MK-7264 (formerly AF-219), which significantly inhibited daytime objective cough frequency by $75 \%$. This indicates that the 
endogenous ligand of this ion channel, ATP, is likely to play a role in refractory chronic cough [78]. Two clinical studies highlighted at the ERS Congress showed further results with this compound. SMITH et al. [79] presented the results of a double-site, 12-week parallel group study with MK-7264 in patients with refractory chronic cough. A total of 253 patients were given a placebo or the P2X3 antagonist MK-7264 (one of three doses: $7.5 \mathrm{mg}, 20 \mathrm{mg}$ or $50 \mathrm{mg}$, all of which were lower than that used in the original study where most patients experienced dysgeusia) and awake cough frequency and 24-h cough frequency were assessed. Despite a large placebo effect, both of these end points were significantly reduced following treatment with the $50 \mathrm{mg}$ dose of MK-7264, indicating that this compound is efficacious at more well-tolerated doses. Morice et al. [80] also presented a study utilising this compound. Their study looked at evoked tussive responses to ATP, citric acid, capsaicin or distilled water (fog) following treatment with MK-7264 or a placebo in healthy controls and patients with refractory chronic cough. All tussive challenges evoked a cough response in chronic cough patients but only cough responses to ATP and fog were inhibited with the antagonist, indicating successful target engagement by the compound. Another study indicated that activation of airway sensory nerves by hypo-osmolar solutions such as distilled water was through activation of the ion channels TRPV4 and TRPM3, which release ATP to activate P2X3 [81].

Other notable studies investigated other receptors which may be involved in the cough response. DicPinigatis et al. [82] looked at the effect of the $\alpha 7$ nicotinic receptor in guinea pig cough. The authors observed that in healthy non-smoking individuals, e-cigarettes containing nicotine had a marked antitussive effect and therefore hypothesised that nicotine could inhibit cough. In a guinea pig model, it was indicated that nicotine and selective agonists of the $\alpha 7$ nicotinic receptor inhibited cough induced by citric acid and therefore the antitussive effects of e-cigarettes were likely to be through the $\alpha 7$ nicotinic receptor. A further study by WORTLEY et al. [83] highlighted the inhibitory effects of agonists of the EP2 and EP4 receptors on TRPV1 induced responses. EP2 and EP4 are receptors for the inflammatory mediator prostaglandin E2, which has been shown to have a variety of beneficial and detrimental effects in the airways. EP3 evokes cough and EP2 and EP4 have protective bronchodilator and anti-inflammatory effects; however, these inhibitory effects are lost following cigarette smoke exposure indicating that prostanoids could play a role in cough in diseases such as COPD.

Overall, the novel studies showcased at the ERS Congress indicate that the search for an effective treatment for the troublesome problem of chronic refractory cough is underway. Perhaps the most promising is the P2X3 antagonist MK-7264, which has now shown efficacy in two clinical trials along with successful target engagement. In addition, further pre-clinical studies have highlighted other potential targets for the treatment of refractory chronic cough.

\section{References}

$1 \quad$ Pavord ID, Bush A, Holgate S. Asthma diagnosis: addressing the challenges. Lancet Respir Med 2015; 3: 339-341. Wenzel SE. Asthma: defining of the persistent adult phenotypes. Lancet 2006; 368: 804-813.

Accordini S, Corsico AG, Braggion $\mathrm{M}$, et al. The cost of persistent asthma in Europe: an international population-based study in adults. Int Arch Allergy Immunol 2013; 160: 93-101.

4 Koczulla AR, Vogelmeier CF, Garn H, et al. New concepts in asthma: clinical phenotypes and pathophysiological mechanisms. Drug Discov Today 2017; 22: 388-396.

5 Chung KF, Adcock IM. How variability in clinical phenotypes should guide research into disease mechanisms in asthma. Ann Am Thorac Soc 2013; 10: Suppl., S109-S117.

6 Bigler J, Boedigheimer M, Schofield JPR, et al. A severe asthma disease signature from gene expression profiling of peripheral blood from U-BIOPRED cohorts. Am J Respir Crit Care Med 2017; 195: 1311-1320.

7 Affleck K, Taylor A, Riley JH, et al. Late breaking abstract - comparison of the blood transcriptomic profiles of adults and children from the U-BIOPRED asthma study. Eur Respir J 2017; 50: Suppl. 61, OA3442.

8 Amelink M, de Nijs SB, de Groot JC, et al. Three phenotypes of adult-onset asthma. Allergy 2013; 68: 674-680.

9 Hekking P-P, Loza MJ, Pavlidis S, et al. Pathway discovery using transcriptomic profiles in adult-onset severe asthma. J Allergy Clin Immunol 2017; in press [https:/doi.org/10.1016/j.jaci.2017.06.037].

10 Grainge CL, Davies DE. Epithelial injury and repair in airways diseases. Chest 2013; 144: 1906-1912.

11 Pavlidis S, Guo Y, Sun K, et al. Molecular evidence of Group 1 innate lymphoid cell activation in the U-BIOPRED cohort. Eur Respir J 2017; 50: Suppl. 61, OA278.

12 Farne HA, Johnston SL. Immune mechanisms of respiratory viral infections in asthma. Curr Opin Immunol 2017; 48: 31-37.

13 Johnston SL, Pattemore PK, Sanderson G, et al. Community study of role of viral infections in exacerbations of asthma in 9-11 year old children. BMJ 1995; 310: 1225-1229.

14 Bizzintino J, Lee WM, Laing IA, et al. Association between human rhinovirus C and severity of acute asthma in children. Eur Respir J 2011; 37: 1037-1042.

15 Johnston SL, Pattemore PK, Sanderson G, et al. The relationship between upper respiratory infections and hospital admissions for asthma: a time-trend analysis. Am J Respir Crit Care Med 1996; 154: 654-660.

16 Jackson DJ, Lemanske RF Jr. The role of respiratory virus infections in childhood asthma inception. Immunol Allergy Clin North Am 2010; 30: 513-522.

17 Sigurs N, Gustafsson PM, Bjarnason R, et al. Severe respiratory syncytial virus bronchiolitis in infancy and asthma and allergy at age 13. Am J Respir Crit Care Med 2005; 171: 137-141. 

influences viral infections and illness. I Allergy Clin Immunol 2017; 140: 909-920.

19 Holt PG, Sly PD. Interaction between adaptive and innate immune pathways in the pathogenesis of atopic asthm operation of a lung/bone marrow axis. Chest 2011; 139: 1165-1171.

20 Rubner FJ, Jackson DJ, Evans MD, et al. Early life rhinovirus wheezing, allergic sensitization, and asthma risk at adolescence. J Allergy Clin Immunol 2017; 139: 501-507.

21 Toussaint M, Jackson DJ, Swieboda D, et al. Host DNA released by NETosis promotes rhinovirus-induced type-2 allergic asthma exacerbation. Nature Med 2017; 23: 681-691.

22 Danov O, Laßwitz L, Obernolte H, et al. Ex vivo virus infection of HDM-sensitized lung tissue proves inadequate anti-viral immune response in small airways. Eur Respir J 2017; 50: Suppl. 61, PA1140.

23 Lin J, Zhao GQ, Wang Q, et al. Regulation of interleukin 33/ST2 signaling of human corneal epithelium in allergic diseases. Int J Ophthalmol 2013; 6: 23-29.

24 Moffatt MF, Gut IG, Demenais F, et al. A large-scale, consortium-based genome-wide association study of asthma. N Engl J Med 2010; 363: 1211-1221.

25 Ravanetti L, Dijkhuis A, Sabogal Pineros Y, et al. Late breaking abstract - IL-33 drives influenza-induced exacerbations of asthma by halting anti-viral and Th1 responses. Eur Respir J 2017; 50: Suppl. 61, PA1138.

26 Haccuria A, Virreira M, Rascchaert J, et al. IL-33 mRNA in induced sputum cells from allergic rhinitis and asthma patients: a marker of Th-2 phenotype status? Eur Respir J 2017; 50: Suppl. 61, PA4767.

27 Perkins T, Oczypok E, Dutz R, et al. RAGE-dependent VCAM-1 expression in the pulmonary endothelium mediates IL-33 induced asthma pathogenesis. Eur Respir J 2017; 50: Suppl. 61, PA4917.

28 Prefontaine D, Lajoie-Kadoch S, Foley S, et al. Increased expression of IL-33 in severe asthma: evidence of expression by airway smooth muscle cells. J Immunol 2009; 183: 5094-5103.

29 Kamekura R, Kojima T, Takano K, et al. The role of IL-33 and its receptor ST2 in human nasal epithelium with allergic rhinitis. Clin Exp Allergy 2012; 42: 218-228.

30 Mizutani N, Nabe T, Yoshino S. Interleukin-33 and alveolar macrophages contribute to the mechanisms underlying the exacerbation of IgE-mediated airway inflammation and remodelling in mice. Immunology 2013; 139: 205-218.

31 Lynch JP, Werder RB, Simpson J, et al. Aeroallergen-induced IL-33 predisposes to respiratory virus-induced asthma by dampening antiviral immunity. J Allergy Clin Immunol 2016; 138: 1326-1337.

32 Werder RB, Zhang V, Lynch JP, et al. Chronic IL-33 expression predisposes to viral-induced exacerbations of asthma by increasing type 2 inflammation and dampening antiviral immunity. J Allergy Clin Immunol 2017; in press [https://doi.org/10.1016/j.jaci.2017.07.051].

33 Upham, J, Jurak L, Xi Y. IL-33 augments rhinovirus-induced type 2 immune responses in asthma via selective upregulation of one chain of the IL-33 receptor. Eur Respir J 2017; 50: Suppl. 61, PA567.

34 Vogelmeier CF, Criner GJ, Martinez FJ, et al. Global Strategy for the Diagnosis, Management, and Prevention of Chronic Obstructive Lung Disease 2017 Report: GOLD Executive Summary. Eur Respir J 2017; 49 : 1700214.

35 Lipson DA, Barnacle H, Birk R, et al. FULFIL Trial: once-daily triple therapy for patients with chronic obstructive pulmonary disease. Am J Respir Crit Care Med 2017; 196: 438-446.

36 Naya I, Barnacle H, Birk R, et al. Clinically important deterioration in advanced COPD patients using single inhaler triple therapy: results from the FULFIL study. Eur Respir J 2017; 50: Suppl. 61, PA3248.

37 Vestbo J, Papi A, Corradi M, et al. Single inhaler extrafine triple therapy versus long-acting muscarinic antagonist therapy for chronic obstructive pulmonary disease (TRINITY): a double-blind, parallel group, randomised controlled trial. Lancet 2017; 389: 1919-1929.

38 Singh D, Papi A, Vezzoli S, et al. Effect of CHF5993 pMDI (extrafine beclometasone dipropionate: BDP, formoterol fumarate: FF, glycopyrronium bromide: GB) on clinically important deteriorations (CID) in COPD post-hoc analysis of TRINITY study. Eur Respir J 2017; 50: Suppl. 61, PA3949.

39 Singh D, Papi A, Corradi M, et al. Single inhaler triple therapy versus inhaled corticosteroid plus long-acting $\beta_{2}$-agonist therapy for chronic obstructive pulmonary disease (TRILOGY): a double-blind, parallel group, randomised controlled trial. Lancet 2016; 388: 963-973

40 Singh D, Fabbri L, Pappi A, et al. Late breaking abstract - extrafine triple therapy reduces exacerbations in GOLD B COPD patients: post-hoc analysis of TRILOGY and TRINITY. Eur Respir J 2017; 50: Suppl. 61, OA2898.

41 Wedzicha JA, Banerji D, Chapman KR, et al. Indacaterol-Glycopyrronium versus Salmeterol-Fluticasone for COPD. N Engl J Med 2016; 374: 2222-2234.

42 Bjermer L, Roche N, Gutzwiller FS, et al. Estimating the number of patients' needed to treat (NNT) with indacaterol/glycopyrronium to prevent COPD exacerbations: results from the FLAME study. Eur Respir J 2017; 50: Suppl. 61, PA936.

43 Anzueto, AR, Kostikas K, Shen S, et al. Indacaterol/glycopyrronium (IND/GLY) reduces the risk of clinically important deterioration (CID) versus salmeterol/fluticasone (SFC): the FLAME study. Eur Respir J 2017; 50: Suppl. 61, PA3255.

44 Wedzicha J, Vogelmeier CF, Ayers T, et al. Respiratory safety profile of indacaterol/glycopyrronium (IND/GLY) versus salmeterol/fluticasone (SFC): results from the FLAME study. Eur Respir J 2017; 50: Suppl. 61, PA1075.

45 Bateman ED, Chapman KR, Singh D, et al. Aclidinium bromide and formoterol fumarate as a fixed-dose combination in COPD: pooled analysis of symptoms and exacerbations from two six-month, multicentre, randomised studies (ACLIFORM2 and AUGMENT). Respir Res 2015; 16: 92.

46 Singh D, D'Urzo AD, Donohue JF, et al. Efficacy of aclidinium/formoterol: pooled post hoc analysis of patients naïve to COPD maintenance therapy. Eur Respir J 2017; 50: Suppl. 61, PA679.

47 Watz H, Troosters T, Beeh KM, et al. ACTIVATE: effect of aclidinium/formoterol on physical activity in patients with COPD. Eur Respir J 2017; 50: Suppl. 61, PA687.

48 Worth H, Buhl R, Criée CP, et al. GOLD 2017 treatment pathways in 'real life': an analysis of the DACCORD observational study. Respir Med 2017; 131: 77-84.

49 Buhl R, Criée C-P, Kardos P, et al. Low rate of exacerbations following initiation of LABA/LAMA fixed-dose combinations: an analysis of the DACCORD real life study. Eur Respir J 2017; 50: Suppl. 61, PA1070.

50 Gosset P, Kone B, Perez-Cruz M, et al. The TLR5 agonist flagellin prevents bacteria-induced chronic obstructive pulmonary disease exacerbations in mice. Eur Respir J 2017; 50: Suppl. 61, OA1490. 
51 To T, Stanojevic S, Moores G, et al. Global asthma prevalence in adults: findings from the cross-sectional world health survey. BMC Public Health 2012; 12: 204.

52 Adeloye D, Chua S, Lee C, et al. Global and regional estimates of COPD prevalence: systematic review and meta-analysis. J Glob Health 2015; 5: 020415.

53 Chung KF, Wenzel SE, Brozek JL, et al. International ERS/ATS guidelines on definition, evaluation and treatment of severe asthma. Eur Respir J 2014; 43: 343-373.

54 Vestbo J, Papi A, Corradi M, et al. Single inhaler extrafine triple therapy versus long-acting muscarinic antagonist therapy for chronic obstructive pulmonary disease (TRINITY): a double-blind, parallel group, randomised controlled trial. Lancet 2017; 389: 1919-1929.

55 Bafadhel M, Davies L, Calverley PM, et al. Blood eosinophil guided prednisolone therapy for exacerbations of COPD: a further analysis. Eur Respir J 2014; 44: 789-791.

56 Gauvreau GM, O'Byrne PM, Boulet LP, et al. Effects of an anti-TSLP antibody on allergen-induced asthmatic responses. N Engl J Med 2014; 370: 2102-2110.

57 Chen R, Smith SG, Salter B, et al. Allergen-induced increases in sputum levels of Group 2 innate lymphoid cells in subjects with asthma. Am J Respir Crit Care Med 2017; 196: 700-712.

58 Corren J, Parnes JR, Wang L, et al. Tezepelumab in adults with uncontrolled asthma. N Engl J Med 2017; 377: 936-946.

59 Siva R, Green RH, Brightling CE, et al. Eosinophilic airway inflammation and exacerbations of COPD: a randomised controlled trial. Eur Respir J 2007; 29: 906-913.

60 Pavord ID, Chanez P, Criner GJ, et al. Mepolizumab for eosinophilic chronic obstructive pulmonary disease. N Engl J Med 2017; 377: 1613-1629.

61 Chalmers JD, Finch S, Shteinberg M, et al. Late breaking abstract - the prevalence and burden of Pseudomonas aeruginosa among bronchiectasis patients in Europe - data from the FRIENDS cohort. Eur Respir J 2017; 50: Suppl. 61, OA1967.

62 Smith A, Choi J-Y, Finch S, et al. Sputum pregnancy zone protein (PZP) - a potential biomarker of bronchiectasis severity. Eur Respir J 2017; 50: Suppl. 61, OA1969.

63 Froehlich J, Dahms J, Cipolla D, et al. Late breaking abstract - reduction in frequency of pulmonary exacerbations (PE) with inhaled ARD-3150 in non-cystic fibrosis bronchiectasis (NCFB) patients is independent of Pseudomonas aeruginosa (PA) susceptibility at baseline. Eur Respir J 2017; 50: Suppl. 61, OA466.

64 Kanoh S, Rubin BK. Mechanisms of action and clinical application of macrolides as immunomodulatory medications. Clin Microbiol Rev 2010; 23: 590-615.

65 Menzel M, Akbarshahi H, Bjermer L, et al. Azithromycin induces anti-viral effects in cultured bronchial epithelial cells from COPD patients. Sci Rep 2016; 6: 28698.

$66 \mathrm{Wu} \mathrm{Q}$, Shen W, Cheng H, et al. Long-term macrolides for non-cystic fibrosis bronchiectasis: a systematic review and meta-analysis. Respirology 2014; 19: 321-329.

67 Altenburg J, de Graaff CS, Stienstra Y, et al. Effect of azithromycin maintenance treatment on infectious exacerbations among patients with non-cystic fibrosis bronchiectasis: the BAT randomized controlled trial. JAMA 2013; 309: 1251-1259.

68 Serisier DJ, Martin ML, McGuckin MA, et al. Effect of long-term, low-dose erythromycin on pulmonary exacerbations among patients with non-cystic fibrosis bronchiectasis: the BLESS randomized controlled trial. JAMA 2013; 309: 1260-1267.

69 Valery PC, Morris PS, Byrnes CA, et al. Long-term azithromycin for indigenous children with non-cystic-fibrosis bronchiectasis or chronic suppurative lung disease (bronchiectasis intervention study): a multicentre, double-blind, randomised controlled trial. Lancet Respir Med 2013; 1: 610-620.

70 Wong C, Jayaram L, Karalus N, et al. Azithromycin for prevention of exacerbations in non-cystic fibrosis bronchiectasis (EMBRACE): a randomised, double-blind, placebo-controlled trial. Lancet 2012; 380: 660-667.

71 Mertsch P, Rademacher J, de Roux A, et al. PROGNOSIS - the German Bronchiectasis Registry: first results. Eur Respir J 2017; 50: Suppl. 61, PA4059.

72 Dhar R, Chandrashekariah S, Dsouza G, et al. Phenotype characterization of non-cystic fibrosis bronchiectasis in India. Eur Respir J 2017; 50: Suppl. 61, PA4058.

73 Schappert SM, Rechtsteiner EA. Ambulatory medical care utilization estimates for 2007. Vital Heath Statistics 2011; 12: 1-38.

74 Pratter MR. Cough and the common cold: ACCP evidence-based clinical guidelines. Chest 2006; 129: 72S-74S.

75 French CL, Irwin RS, Curley FJ, et al. Impact of chronic cough on quality of life. Arch Intern Med 2010; 158: 1657-1661.

76 Irwin RS, French CT, Lewis SZ, et al. Overview of the management of cough: CHEST guideline and expert panel report. Chest 2014; 146: 885-889.

77 Pacheco A. Chronic cough: from a complex dysfunction of the neurological circuit to the production of persistent cough. Thorax 2014; 69: 881-883.

78 Abdulqawi R, Dockry R, Holt K, et al. P2X3 receptor antagonist (AF-219) in refractory chronic cough: a randomised, double-blind, placebo-controlled phase 2 study. Lancet 2015; 385: 1198-1205.

79 Smith J, Kitt M, Morice A, et al. Inhibition of P2X3 by MK-7264 reduces 24-hour cough frequency in a randomized, controlled, Phase 2b clinical trial. Eur Respir J 2017; 50: Suppl. 61, OA2932.

80 Morice AH, Kitt M, Ford A, et al. The Effect of MK-7264, a P2X3 antagonist, on cough reflex sensitivity in a randomized crossover trial of healthy and chronic cough subjects. Eur Respir J 2017; 50: Suppl. 61, OA2931.

81 Bonvini S, Dubuis E, Adcock J, et al. Activation of transient receptor potential (TRP) channels by hypoosmolar solution: an endogenous mechanism of ATP release and afferent nerve activation. Eur Respir J 2017; 50: Suppl. 61 , OA4410.

82 Dicpinigatis $\mathrm{P}$, Canning B, DeVita R, et al. The antitussive effects of alpha7 ( $\alpha 7)$ nicotinic receptor agonists. Eur Respir J 2017; 50: Suppl. 61, OA4409.

83 Wortley M, Bonvini S, Maher S, et al. EP2 \& EP4 receptor agonists inhibit the airway sensory nerves that cause cough, a potential protective control that is lost in human smokers and a pre-clinical COPD model. Eur Respir $J$ 2017; 50: Suppl. 61, OA1491. 